\title{
Strain Measurement by Local Diffraction: NanoBeam Electron Diffraction (NBED) Compared to Convergent Beam (CBED) and Dark Holography
}

\author{
J.L. Rouvière ${ }^{*}$, A. Béché ${ }^{* *}$, T. Denneulin ${ }^{* * *}$ and D. Cooper ${ }^{* * *}$ \\ * CEA INAC, SP2M, LEMMA Minatec, 17 rue des Martyrs Grenoble, F-38054, France. \\ ** now at FEI Company, Eindhoven, Netherlands \\ *** CEA, LETI, Minatec, 17 rue des Martyrs Grenoble, F-38054, France
}

This presentation will focus on NanoBeam Electron Diffraction and its comparison with Convergent Beam Electron diffraction (CBED) and Dark Field Electron Holography (DFEH). Most experimental results have been obtained in SiGe layers embedded in Si (Fig. 1) or incorporated into transistors (Fig. 2), but some new results on Si nanowires will also be shown. With NBED, a strain sensitivity of $610^{-4}$ on local areas of $3 \times 3 \mathrm{~nm}^{2}$ can be achieved [1]. NBED has several advantages compared to CBED and DFEH. Our conclusion is that with the recent improvements, NBED has become an efficient, simple to use and versatile technique for strain measurement.

The microelectronics industry has been looking for simple tools to quantitatively measure strain in areas at the nanometer scale. In TEM, CBED was first used for that purpose [2]. It consists of acquiring with a small, slightly convergent electron probe a local diffraction pattern (spot diameter much smaller than $1 \mathrm{~nm}$, convergent semi-angle in the order of $10 \mathrm{mrad}$ ). The transmitted beam is the most useful as it contains many line features, called for simplicity HOLZ lines (Fig. 1), and the precise position of these lines allows the strain to be measured with excellent sensitivity $(\Delta \mathrm{a} / \mathrm{a}=$ $2.10^{-4}$ ) in perfect crystals [2]. However, CBED has several limitations. (1) Strain relaxation at interface can create a bending of the planes along the electron beam and this bending produce a broadening of the HOLZ lines [3]. (Fig. 1 c-d). (2) HOLZ lines in the central diffraction spot only appear for thick samples (thickness greater than $100 \mathrm{~nm}$ ). So CBED cannot be applied to nanostructures. (3) In order to obtain fine HOLZ lines, the crystal have to be tilted a few degrees $(\sim$ $10^{\circ}$ ) from a low index zone axis, which can lead to shadowing effects.

More recently, DFEH has been proposed to measured strain [4-5]. The accuracy of this interference technique is tremendous (certainly below $2.10^{-4}$ ), but a reference unstrained area near the region of interest is needed and the sample must be flat with a given thickness (about 100nm).

Alternatively, NBED or NBD (NanoBeam Electron Diffraction) has been used [6]. In this case, local diffraction patterns are acquired along a low index axis, such as $\langle 110\rangle$ in silicon devices, with a parallel beam (convergent semi-angle $<0.5 \mathrm{mrad}$ ) and a beam size larger than in CBED. At first, the technique was limited to beam sizes larger than $10 \mathrm{~nm}$ in diameter and to a strain sensitivity of $10^{-3}$. By using a modern microscope, in our case a FEI TITAN with a probe Cs-corrector, we were able to reduce the spot diameter size to $2.7 \mathrm{~nm}$ with a condenser aperture of $50 \mu \mathrm{m}$ (Fig. 1B) and improve the sensitivity of the technique down to $6.10^{-4}$ (Fig. 1D) [3]. Strain maps (Fig. 2) were also realized (Fig. 2). Applications of NBED is not limited to microelectronics devices. As we will show, any isolated nano-objects, like Si nanowires with a core-shell structure can be studied. The beam shape and the accuracy of NBED will be discussed. 
References

[1] A Béché, J.L. Rouvière, L. Clément et J.M. Hartmann Appl. Phys. Letters 95 ( 2009) 123114

[2] A. Armigliato, R. Balboni, et al., Journal of Applied Physics, 7 (1997) 2375

[3] L. Clement, R. Pantel, et al., Appl. Phys. Letters 85 (2004). 651

[4] M. Hytch, F. Houdellier, F. Hue and E. Snoeck Nature 453 (2008) 7198

[5] A. Béché, J.L. Rouvière, J.P. Barnes and D. Cooper Ultramicroscopy 111( 2011) 227

[6] K. Usuda, T. Mizuno, et al.Applied Surface Science 224, (2004) 113

[7] The European EUREKA/CATRENE program supported this work(CT206 UTTERMOST project)
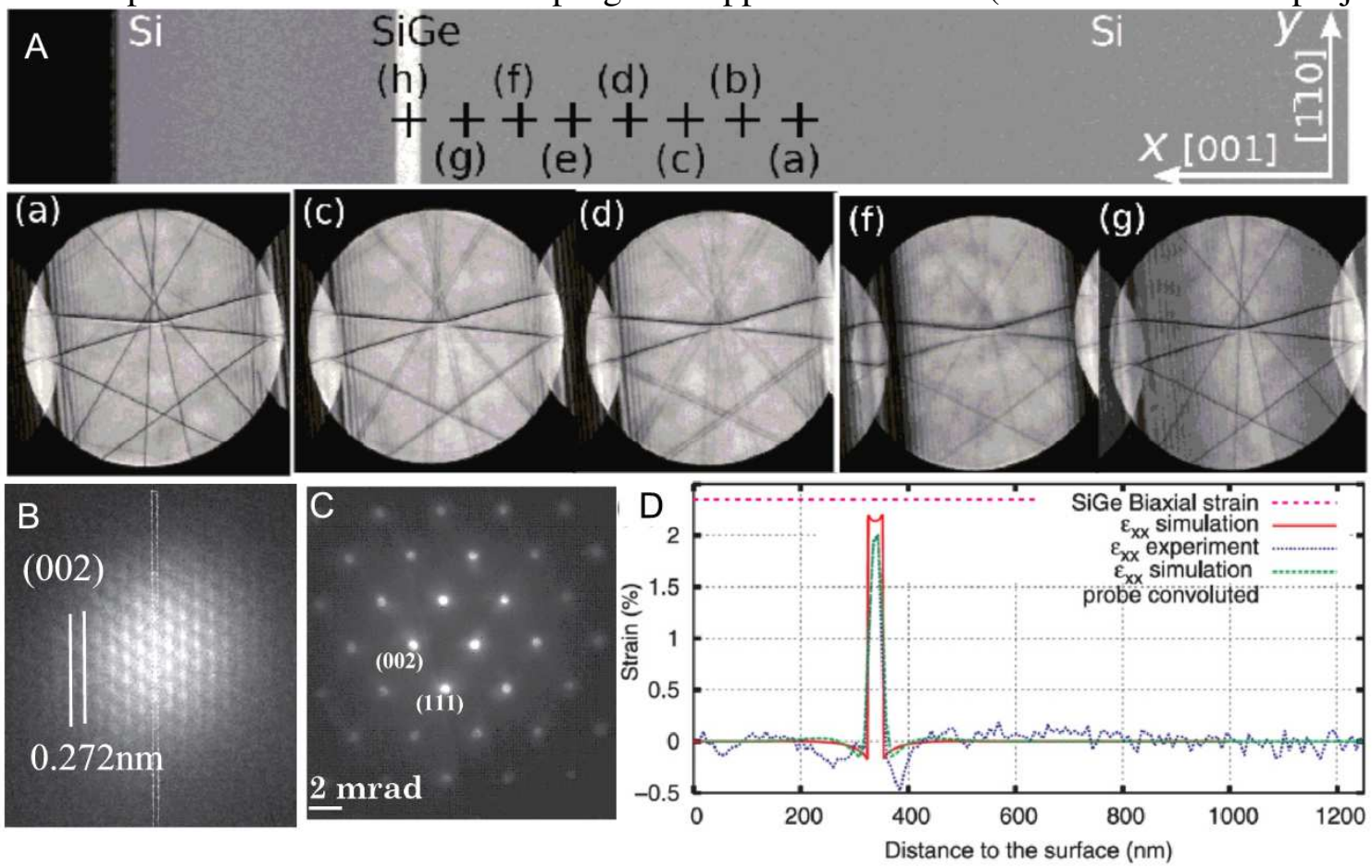

FIG. 1. A) A SiGe/Ge layer embedded in $\mathrm{Si}$ along which either CBED or NBED patterns are acquired. (a-c-d-f-g) Transmitted beam of CBED patterns taken at different distances (marked a-cd-f-g in image A) of the $\mathrm{SiGe} / \mathrm{Si}$ interface B) Image of the NBED probe passing through a $<110>\mathrm{Si}$ crystal. C) Typical NBED pattern acquired in the SiGe/Si sample. D) Strain profile obtained by analyzing the series of NBED patterns. Note that far from the SiGe layer the root mean square in the Si crystal is $6.10^{-4}$ and defines the strain sensitivity of the NBED technique.
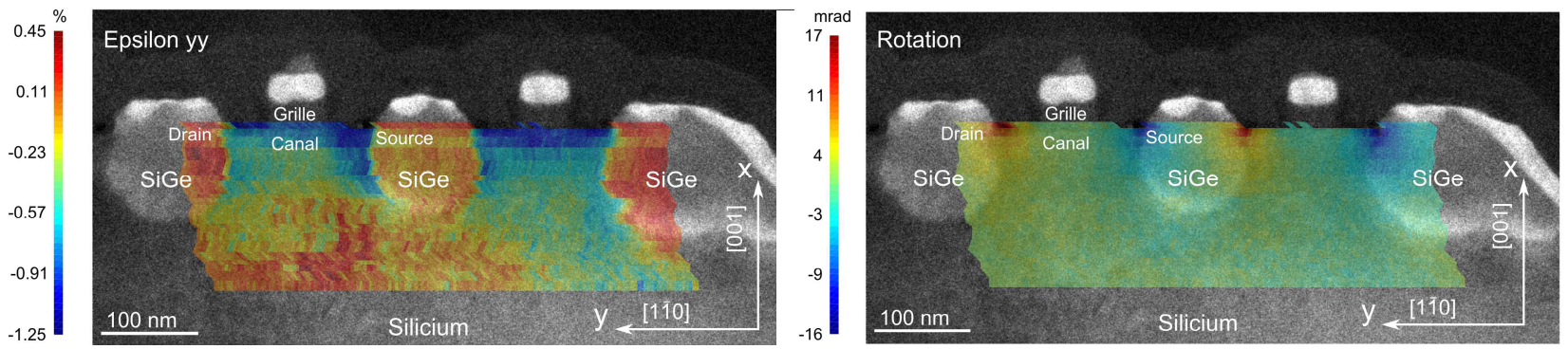

.FIG. 2. Strain maps of a series of transistors.

a) Strain map (epsilon y-y) showing that the Si channel under the gate (named 'grille') is in compression (compression of $-1.2 \%$ ) b) Rotation map showing the rotation of the lattice around the $\mathrm{z}$-axis orthogonal to the $\mathrm{x}$ and $\mathrm{y}$ axis. . 